\title{
Some properties of reachable sets for control affine systems
}

\author{
Marek Grochowski
}

Received: 28 September 2010 / Revised: 11 November 2010 / Accepted: 11 November 2010 /

Published online: 21 December 2010

C The Author(s) 2010. This article is published with open access at Springerlink.com

\begin{abstract}
Let $\dot{q}=X_{0}+\sum_{j=1}^{k} u_{j} X_{j}$ be a control affine system on a manifold $M$, let $C$ be a convex compact subset of $\mathbb{R}^{k}, \operatorname{dim} C>0$, let $q_{0}$ be a fixed point of $M$, and let $U$ be a neighbourhood of $q_{0}$. We consider three reachable sets from $q_{0}$ for our system which are generated by square integrable controls with values in $C, \mathrm{riC}$ - the relative interior of $C$, and $r b C$ - the relative boundary of $C$, respectively, with contraints on a state variable $q$ of the form $q \in U$. Among other things, we investigate the relation between closures, interiors and boundaries of the three reachable sets. We also show how methods of the sub-Lorentzian geometry can serve as an auxiliary tool in the study of control affine systems.
\end{abstract}

Keywords Reachable sets - Affine-control systems - Geometric optimality · Sub-Lorentzian geometry

Mathematics Subject Classification (2000) 34H05 · 93C99

\footnotetext{
M. Grochowski (凶)

Faculty of Mathematics and Science, Cardinal Stefan Wyszyński University, ul. Dewajtis 5, 01-815 Waszaw, Poland e-mail: m.grochowski@uksw.edu.pl; mgrochow@impan.gov.pl
}

M. Grochowski

Institute of Mathematics, Polish Academy of Sciences, ul. Śniadeckich 8, 00-950 Warszaw, Poland 


\section{Introduction and statement of the results}

Consider a control affine system

$$
\dot{q}=X_{0}(q)+\sum_{j=1}^{k} u_{j} X_{j}(q)
$$

where $X_{0}, \ldots, X_{k}$ are smooth linearly independent vector fields defined on a smooth connected manifold $M$, and $\left(u_{1}, \ldots, u_{k}\right) \in C$, where $C$ is a subset of $\mathbb{R}^{k}$. If $q_{0} \in M$ is a fixed point and $U$ is a fixed neighbourhood of $q_{0}$, denote by $\mathcal{A}\left(q_{0}, U ; C\right)$ the set of endpoints of all trajectories of (1.1) that start from $q_{0}$, are generated by square integrable controls $u:[0, T] \longrightarrow C$, and are contained in $U$; here $T>0$ is not fixed. The set $\mathcal{A}\left(q_{0}, U ; C\right)$ is called the reachable set from $q_{0}$ for (1.1) corresponding to the set $C$ of control parameters. Trajectories of (1.1) that are generated by $L^{2}$ controls with values in $C$ will be sometimes referred to as admissible trajectories.

The aim of this paper is to prove Theorem 1.1 which we are going to formulate below. Let $S$ be a set and $\left\{Y_{S}: s \in S\right\}$ be a family of smooth vector fields on $M$. Denote by Lie $\left\{Y_{s}: s \in S\right\}$ the Lie algebra generated by this family. For a $q \in M$ let us also set $\operatorname{Lie}_{q}\left\{Y_{s}: s \in S\right\}=\left\{Z(q): Z \in \operatorname{Lie}\left\{Y_{s}: s \in S\right\}\right\} \subset T_{q} M$. Suppose now that $C \subset \mathbb{R}^{k}$ is a compact and convex set of dimension greater than zero. Thus $C$ is of the form $C=\overline{C_{0}}$, where $C_{0}=r i C$ and $r i$ stands for the interior relative to the affine hull $A f f(C)$ (the so-called relative interior - c.f. [12]). We will also use the notation $r b C$ meaning the boundary of $C$ relative to $A f f(C)$.

Theorem 1.1 Let $C$ be a compact and convex subset of $\mathbb{R}^{k}, \operatorname{dim} C>0$, and let $q_{0} \in M$ be a fixed point. Suppose that the vector fields in (1.1) satisfy

$$
\text { Lie }_{q_{0}}\left\{X_{0}+\sum_{j=1}^{k} u_{j} X_{j}: u \in r b C\right\}=T_{q_{0}} M
$$

Then there exists a fundamental system $\mathcal{O}$ of neighbourhoods of $q_{0}$ such that for every $U \in \mathcal{O}$ the following equalities hold true

$$
\begin{gathered}
\mathcal{A}\left(q_{0}, U ; C\right)=c l_{U}\left(\operatorname{int} \mathcal{A}\left(q_{0}, U ; r i C\right)\right)=c l_{U}\left(\operatorname{int} \mathcal{A}\left(q_{0}, U ; r b C\right)\right), \\
\operatorname{int} \mathcal{A}\left(q_{0}, U ; C\right)=\operatorname{int} \mathcal{A}\left(q_{0}, U ; r i C\right)=\operatorname{int} \mathcal{A}\left(q_{0}, U ; r b C\right), \\
\tilde{\partial} \mathcal{A}\left(q_{0}, U ; C\right)=\tilde{\partial} \mathcal{A}\left(q_{0}, U ; r i C\right)=\tilde{\partial} \mathcal{A}\left(q_{0}, U ; r b C\right) ;
\end{gathered}
$$

here $c l_{U}$ (resp. $\left.\tilde{\partial}\right)$ is the closure (resp. boundary) with respect to $U$.

Remark 1.1 It is useful for further use to notice that the condition (1.2) implies the relation Lie $_{q_{0}}\left\{X_{0}+\sum_{j=1}^{k} u_{j} X_{j}: u \in C\right\}=T_{q_{0}} M$ which in turn implies 
Lie $_{q_{0}}\left\{X_{0}+\sum_{j=1}^{k} u_{j} X_{j}: u \in r i C\right\}=T_{q_{0}}$ M. Clearly, Lie $e_{q_{0}}\left\{X_{0}, X_{1}, \ldots, X_{k}\right\}=$ $T_{q_{0}} M$ can be deduced from (1.2) too.

Keeping the notation from Theorem 1.1 one can also prove, under weaker assumptions, the following

Proposition 1.1 Suppose that $X_{0}, \ldots, X_{k}$ are linearly independent. Then $\mathcal{A}\left(q_{0}, U ; C\right)=c l_{U} \mathcal{A}\left(q_{0}, U ; r i C\right)=c l_{U} \mathcal{A}\left(q_{0}, U ; r b C\right)$ for every $U \in \mathcal{O}$. In particular, the set $\mathcal{A}\left(q_{0}, U ; C\right)$ is closed with respect to $U$. If, moreover, $k \geq 2$ and the distribution Span $\left\{X_{0}, \ldots, X_{k}\right\}$ is generic, then for every open $U$ (not necessarily in $\mathcal{O})$ the set $\mathcal{A}\left(q_{0}, U ;\right.$ riC $)$ is open, and $\mathcal{A}\left(q_{0}, U ; C\right)=\mathcal{A}\left(q_{0}, U ; r b C\right)$.

This moment is suitable for recalling some known facts on reachable sets that can be found in the literature devoted to mathematical control theory (see [1] or eg. [3] and its reference section). First of all, reachable sets for families of smooth vector fields are well described. Such sets, in our situation, correspond to reachable sets for (1.1) generated by piecewise constant controls with values, respectively in $C, r i C, r b C$, and they satisfy, for instance, Krener's theorem (see [10]) which is used later in this paper. It is also known that the formulas (1.4), (1.5) are true with respect to piecewise constant controls (see [9]). Reachable sets generated by piecewise constant controls may differ from ones generated by $L^{2}$ controls, however, both have the same closures.

Now let us focus on $\mathcal{A}\left(q_{0}, U ; C\right)$. As one can see, in order to prove (1.3) in Theorem 1.1 we need to know that $\mathcal{A}\left(q_{0}, U ; C\right)$ is closed relative to $U$. One could try to use the following compactness results. Define $\mathcal{A}\left(q_{0}, U ; C ; T\right)\left(\operatorname{resp} . \mathcal{A}\left(q_{0}, U ; C ; \leq T\right)\right)$ to be the set of endpoints of all trajectories of (1.1) that start from $q_{0}$, are generated by square integrable controls $u:[0, T] \longrightarrow C$ (resp. $u:[0, t] \longrightarrow C$ with $t \leq T$ ), and are contained in $U$. Following eg. [3] we know that $\mathcal{A}\left(q_{0}, U ; C ; T\right)$ is compact, provided that graphs of all trajectories of (1.1) are contained in a compact set $K \subset \mathbb{R} \times U$. This assumption, however, is not satisfied in our case, i.e. for $T=T_{0}$ with $T_{0}$ determined as in Proposition 2.1 and Corollary 2.1 below. One could also try to apply the fact that by [9] $\mathcal{A}\left(q_{0}, U ; M ; \leq T\right)$ is compact, provided that for every $u \in L^{2}([0, T], C)$ the trajectory of (1.1) starting from $q_{0}$ and generated by $u$ is defined on the whole $[0, T]$. Again this assumption, with $T=T_{0}$ and $M$ replaced by $U$, is not true in our case. Moreover, $\mathcal{A}\left(q_{0}, U ; C ; \leq T\right) \neq \mathcal{A}\left(q_{0}, M ; C ; \leq T\right) \cap U$. All this permits us to conclude that closeness of $\mathcal{A}\left(q_{0}, U ; C\right)$ requires a separate proof. At the end recall (see again [3]) that $c l_{U} \mathcal{A}\left(q_{0}, U ; C\right)=c l_{U} \mathcal{A}\left(q_{0}, U ; r i C\right)=c l_{U} \mathcal{A}\left(q_{0}, U ; r b C\right)$, the fact which will be used later. Summing up all what we have said, there is a subtal difference between our results and those that can be found in the literature.

Remark at the end of this section that a very interesting situation arises when $C=$ $\left\{u \in \mathbb{R}^{k}:|u| \leq 1\right\}$. Then, as it was noticed in [7], the system (1.1) becomes part of the sub-Lorentzian geometry. In particular, reachable sets from $q_{0}$ for $(1.1)$, for this specific $C$, coincide with the corresponding reachable sets $J^{+}\left(q_{0}, U\right), I^{+}\left(q_{0}, U\right), N^{+}\left(q_{0}, U\right)$ for the sub-Lorentzian structure $(H, g)$ defined as follows: $H=\operatorname{Span}\left\{X_{0}, \ldots, X_{k}\right\}$, $g\left(X_{0}, X_{0}\right)=-1, g\left(X_{j}, X_{j}\right)=+1, j=1, \ldots, k, g\left(X_{\alpha}, X_{\beta}\right)=0, \alpha, \beta=0, \ldots, k$, $\alpha \neq \beta$, and $X_{0}$ is a time orientation. The reasoning is as follows. Obviously, every trajectory of (1.1) is a nonspacelike future directed curve with respect to $(H, g)$. Conversely, suppose that $\gamma:[0, T] \longrightarrow U$ is nonspacelike future directed, 
i.e. $\frac{d}{d t} \gamma(t)=\sum_{a=0}^{k} u_{\alpha}(t) X_{\alpha}(\gamma(t)),-u_{0}^{2}(t)+\sum_{j=1}^{k} u_{j}^{2}(t) \leq 0$. Without loss of generality one can assume that $u_{0}(t)>0$ a.e. Now suitable reparameterization $t=t(s)$ gives $\frac{d}{d s}\left(\gamma(t(s))=X_{0}(\gamma(t(s)))+\sum_{j=1}^{k} \frac{u_{j}(t(s))}{u_{0}(t(s))} X_{j}(\gamma(t(s)))\right.$. This implies $\mathcal{A}\left(q_{0}, U ; C\right)=J^{+}\left(q_{0}, U\right)$ and similarly for the other reachable sets.

Note that (1.3), (1.4), (1.5) have been already obtained in [7] in the sub-Lorentzian setting. Thus the present paper can be viewed as a generalization of [7].

\section{Proof of Theorem 1.1}

This section is devoted to proving Theorem 1.1. By the way we will state some partial results. Below we will need some notions and facts from the sub-Lorentzian geometry, such as a sub-Lorentzian metric on a manifold, a time orientation of a sub-Lorentzian metric, and also the sub-Lorentzian distance from a given point $q_{0}$, normal neighbourhoods of $q_{0}$, the horizontal gradient of a smooth function, and the time-like reachable set from $q_{0}$, each induced by a given sub-Lorentzian metric. For all these notions, their properties and correponding results the reader is referred to [5], [6], [7].

Throughout this section $C$ will be a compact convex subset of $\mathbb{R}^{k}, \operatorname{dim} C>0$. At the begining let us assume that the fields $X_{0}, \ldots, X_{k}$ in (1.1) are linearly independent. Unless otherwise stated all controls are supposed to be square integrable with values in $C$.

Our first aim is to determine a fundamental system of neighbourhoods of a given point $q_{0} \in M$ from the hypothesis of Theorem 1.1 To this end let $\hat{U}$ be a neighbourhood of $q_{0}$. One can find a function $\varphi \in C^{\infty}(\hat{U})$ such that $X_{0}(\varphi)\left(q_{0}\right)=1, X_{j}(\varphi)\left(q_{0}\right)=0$, $j=1, \ldots, k$. Of course such a $\varphi$ is not uniquely determined.

From now on we suppose that $\hat{U}$ is bounded and small enough to have

$$
\inf \left\{X_{0}(\varphi)(q): q \in \hat{U}\right\}>k c \sup \left\{\left|X_{j}(\varphi)(q)\right|: q \in \hat{U}, j=1, \ldots, k\right\}
$$

where $c=\max \left\{\left|u_{j}\right|: j=1, \ldots, k,\left(u_{1}, \ldots, u_{k}\right) \in C\right\}$. We define a sub-Lorentzian time-oriented metric on $\hat{U}$ in the following manner: $H=\operatorname{Span}\left\{X_{0}, \ldots, X_{k}\right\}$ and

$$
\begin{aligned}
& g\left(X_{0}, X_{0}\right)=-k c^{2}-\varepsilon, \quad g\left(X_{j}, X_{j}\right)=+1, \quad j=1, \ldots, k \\
& g\left(X_{\alpha}, X_{\beta}\right)=0, \quad \alpha \neq \beta, \quad \alpha, \beta=0, \ldots, k
\end{aligned}
$$

where $\varepsilon>0$, and $X_{0}$ is a time orientation. In other words the family

$$
\frac{1}{\sqrt{k c^{2}+\varepsilon}} X_{0}, X_{1}, \ldots, X_{k}
$$

is an orthonormal frame for $g$. Let $\gamma:[0, T] \longrightarrow \hat{U}, \gamma(0)=q_{0}$, be an admissible trajectory generated by a control $\left(u_{1}, \ldots, u_{k}\right):[0, T] \longrightarrow C$. Clearly, by (2.2), $g(\dot{\gamma}(t), \dot{\gamma}(t))=-k c^{2}-\varepsilon+\sum_{j=1}^{k} u_{j}^{2}(t) \leq-k c^{2}-\varepsilon+k c^{2}=-\varepsilon<0$ for almost every $t \in[0, T]$. Thus trajectories of (1.1) which are contained in $\hat{U}$ are timelike 
future directed curves with respect to the structure $(H, g)$. By the way we obtain $\mathcal{A}\left(q_{0}, \hat{U} ; C\right) \subset I^{+}\left(q_{0}, \hat{U}\right)$.

Now, after shrinking $\hat{U}$, we suppose that $\hat{U}$ is contained in a normal, relative to $(H, g)$, neighbourhood of a point $q_{0}$. For such a $\hat{U}$ we can prove the following proposition.

Proposition 2.1 There exists a constant $T_{0}>0$ with the following property: if $\gamma$ : $[0, T] \longrightarrow \hat{U}, \gamma(0)=q_{0}$, is an arbitrary trajectory of $(1.1)$ then $T \leq T_{0}$.

Proof Denote by $f[V]$ the sub-Lorentzian distance from $q_{0}$ relative to $V$, where $V$ is a normal neighbourhood of $q_{0}$ containing $\hat{U}$. It is known [5] that $f[V]$ is bounded on $V$ by a positive constant, say, $A$. Therefore if $\gamma:[0, T] \longrightarrow \hat{U}$ is a trajectory of (1.1) starting from $\gamma(0)=q_{0}$ then $L(\gamma) \leq f[V](\gamma(T)) \leq A$, where $L(\gamma)$ is the sub-Lorentzian length of $\gamma$. However,

$$
L(\gamma)=\int_{0}^{T} \sqrt{-g(\dot{\gamma}(t), \dot{\gamma}(t))} d t \geq T \sqrt{\varepsilon},
$$

so it is enough to define $T_{0}=\frac{A}{\sqrt{\varepsilon}}$ and the proof is over.

Note that $A$ increases along with $\varepsilon$, so substituting for $\varepsilon$ bigger and bigger numbers does not cause $T_{0}$ to become smaller. Using Proposition 2.1 we immediately obtain

Corollary 2.1 For any sufficiently small neighbourhood $\hat{U}$ of $q_{0}$ there exist a number $T>0$ such that

$$
\mathcal{A}\left(q_{0}, \hat{U} ; C\right)=\mathcal{A}\left(q_{0}, \hat{U} ; C ; \leq T\right) .
$$

Proceeding further let us remark that if $\gamma:[0, T] \longrightarrow \hat{U}$ is a trajectory of (1.1), the function $t \longrightarrow \varphi(\gamma(t))$ is increasing. Indeed, by (2.1) we have

$$
\frac{d}{d t}\left(\varphi(\gamma(t))=X_{0}(\varphi)(\gamma(t))+\sum_{j=1}^{k} u_{j}(t) X_{j}(\varphi)(\gamma(t))>0 .\right.
$$

In the sub-Lorentzian language, this last remark can be explained by observing that the horizontal gradient $\nabla_{H} \varphi$ is timelike past directed, so $\varphi$ must increase along timelike future directed curves. Now, let $U$ be a neighbourhood of $q_{0}$ such that $U$ is starshaped about $q_{0}, U \subset \hat{U}$, and $\mathcal{A}\left(q_{0}, \hat{U} ; C\right) \cap \partial U=\{\varphi=$ const $\}$ for some const $>\varphi\left(q_{0}\right)$. Obviously, for any $\hat{U}$ one can construct a $U$ with above properties, hence sets of type $U$ form a fundamental system of neighbourhoods of $q_{0}$ which we will denote by $\mathcal{O}$. We shall see below that after slight modification this system of neighbourhoods of $q_{0}$ is sufficient to prove Theorem 1.1

In the sequel we assume that $U$ is a fixed element of the family $\mathcal{O}$. Let us note an easy. 
Corollary 2.2 Let $\gamma:[0, T] \longrightarrow M$ be a trajectory of (1.1) with $\gamma(0) \in U$. If $\gamma\left(t_{0}\right) \in \partial U$ for a $t_{0} \in(0, T)$ then there exists an $\varepsilon>0$ such that $\gamma(t) \notin \bar{U}$ for every $t \in\left(t_{0}, t_{0}+\varepsilon\right)$.

We will need a notion of convergence which is suitable for admissible trajectories. The most useful is a concept of $C^{0}$ convergence which is extensively used eg. in the Lorentzian geometry. More precisely let $\eta_{v}:\left[a_{v}, b_{v}\right] \longrightarrow M, v=1,2, \ldots$, and $\eta:[a, b] \longrightarrow M$ be curves in $M$. We say that a sequence $\left\{\eta_{\nu}\right\}$ tends to $\eta$ in the $C^{0}$ topology on curves if $\eta_{\nu}\left(a_{v}\right) \longrightarrow \eta(a), \eta_{v}\left(b_{v}\right) \longrightarrow \eta(b)$ as $v \longrightarrow \infty$ and for every neighbourhood $V$ of the set $\eta([a, b])$ there exists a number $\Lambda>0$ such that $\eta_{\nu}\left(\left[a_{\nu}, b_{\nu}\right]\right) \subset V$ for every $v>\Lambda$.

Before proving the main result concerning sequences of admissible trajectories we will prove a simple lemma. Remark that if $v \in L^{2}\left([a, b], \mathbb{R}^{k}\right)$ and $F \subset \mathbb{R}^{k}$ then the notation $v([a, b]) \subset F$ means that $v(t) \in F$ for almost every $t \in[a, b]$. Here and below we make no distinction in notation between a sequence and its subsequences.

Lemma 2.1 Let $u^{(v)}, u \in L^{2}\left([a, b], \mathbb{R}^{k}\right), v=1,2, \ldots$, be such that $u^{(v)} \longrightarrow u$ weakly in $L^{2}$. Suppose moreover that $u^{(v)}([a, b]) \subset C$ for every $v$. Then also $u([a, b]) \subset C$.

Proof Let $W=\left\{v \in L^{2}\left([a, b], \mathbb{R}^{k}\right): v([a, b]) \subset C\right\}$. Since $C$ is convex, $W$ is a convex subset of $L^{2}\left([a, b], \mathbb{R}^{k}\right)$. It is known [8] that convex sets are weakly closed if and only if they are closed, therefore it is enough to observe that $W$ is a closed subset of $L^{2}\left([a, b], \mathbb{R}^{k}\right)$ in $L^{2}$ topology. To this end take a sequence $v^{(v)}$ of elements of $W$ which is $L^{2}$-convergent to a $v \in L^{2}\left([a, b], \mathbb{R}^{k}\right)$. Then, after passing to a subsequence, $v^{(v)} \longrightarrow v$ a.e. on $[a, b]$. Since $C$ is closed, it follows that $v([a, b]) \subset C$.

It will be convenient to introduce the following notation. Let $h \in L^{2}\left([0, T], \mathbb{R}^{k+1}\right)$; denote by $\Phi_{t}^{h}$ the flow of non-autonomous vector field $Z(t, q)=\sum_{\alpha=0}^{k} h_{\alpha}(t) X_{\alpha}(q)$ computed starting from $t=0$. In other words the curve $[0, T] \ni t \longrightarrow \Phi_{t}^{h}\left(q_{0}\right)$ is the solution to the Cauchy problem

$$
\dot{q}=Z(t, q), \quad q(0)=q_{0}
$$

For instance, if $u:[0, T] \longrightarrow C$ is a control then $t \longrightarrow \Phi_{t}^{(1, u)}\left(q_{0}\right)$ is the corresponding trajectory of (1.1) initiating at $q_{0}$.

The proposition below, which is a little bit too strong for our purposes, is of its own interest.

Proposition 2.2 Let $\gamma_{v}:\left[0, l_{v}\right] \longrightarrow U$ be a sequence of trajectories of (1.1) such that $\gamma_{v}(0)=q_{0}, v=1,2, \ldots$, and $\gamma_{v}\left(l_{v}\right) \longrightarrow q$ as $v \longrightarrow \infty$ with $q \in U$. Then, after passing to a subsequence, $\gamma_{v} \longrightarrow \gamma$ in the $C^{0}$ topology on curves, where $\gamma:[0, l] \longrightarrow U$ is a trajectory of $(1.1), \gamma(0)=q_{0}, \gamma(l)=q$.

Proof We know by Proposition 2.1 that there exists a constant $T_{0}>0$ such that $l_{v} \leq T_{0}$. Thus, passing to a subsequence if necessary, we may assume that $\left\{l_{v}\right\}$ converges to a number $l$, and either $l_{v} \nearrow l$ or $l_{v} \searrow l$.

Suppose that $l_{v} \nearrow l$. Let $\gamma_{v}(t)=\Phi_{t}^{\left(1, u^{(v)}\right)}\left(q_{0}\right), 0 \leq t \leq l_{v}$, where $u^{(v)}$ is a control generating $\gamma_{v}$. For every $v$ sufficiently large, $u^{(v)}$ can be extended to a control 
$\hat{u}^{(v)}:[0, l] \longrightarrow C$ in such a way that $\hat{\gamma}_{v}(t)=\Phi_{t}^{\left(1, \hat{u}^{(v)}\right)}\left(q_{0}\right)$ does not leave $U$ for $t \in[0, l]$. Obviously $\left\|\hat{u}^{(v)}\right\|_{L^{2}} \leq c \sqrt{k l}$ for every $v$, so again passing to a subsequence we may suppose that there exists a $u \in L^{2}\left([0, l], \mathbb{R}^{k}\right)$ such that $\left(1, \hat{u}^{(v)}\right) \longrightarrow(1, u)$ weakly in $L^{2}$. Now if $\gamma(t)=\Phi_{t}^{(1, u)}\left(q_{0}\right), 0 \leq t \leq l$, then $\hat{\gamma}_{v} \rightrightarrows \gamma$ on $[0, l]$ as $v \longrightarrow \infty$ by properties of flows of type $\Phi_{t}^{h}(\cdot)$ (see [2]). Since $u([0, l]) \subset C$ by Lemma 2.1, $\gamma$ is a trajectory of (1.1) and, by Corollary 2.2, $\gamma$ does not leave $U$. Further $\hat{\gamma}_{v}$ tends to $\gamma$ continuously [11], i.e. $\gamma_{v}\left(l_{v}\right)=\hat{\gamma}_{v}\left(l_{v}\right) \longrightarrow \gamma(l), v \longrightarrow \infty$. Now it is clear that $\gamma_{v} \longrightarrow \gamma$ in the $C^{0}$ topology on curves.

Suppose now that $l_{v} \searrow l$. Take a small $\varepsilon>0$. For every $v$ sufficiently large $l_{v} \leq$ $l+\varepsilon$. If $\varepsilon$ is small enough (and $v$ 's are sufficiently large), every $u^{(v)}$ can be extended to a control $\hat{u}^{(v)}:[0, l+\varepsilon] \longrightarrow C$ in such a way that $\hat{\gamma}_{v}(t)=\Phi_{t}^{\left(1, \hat{u}^{(v)}\right)}\left(q_{0}\right)$ does not leave $U$ for $t \in[0, l+\varepsilon]$. Analogously as in the first case there exists a $\hat{u} \in L^{2}\left([0, l+\varepsilon], \mathbb{R}^{k}\right)$ such that, possibly after passing to a subsequence, $\left(1, \hat{u}^{(v)}\right) \longrightarrow(1, \hat{u})$ weakly in $L^{2}$. Now, if $\hat{\gamma}(t)=\Phi_{t}^{(1, \hat{u})}\left(q_{0}\right), 0 \leq t \leq l+\varepsilon$, then

$$
\hat{\gamma}_{\nu} \rightrightarrows \hat{\gamma} \quad \text { on }[0, l+\varepsilon]
$$

and, since this convergence is continuous, $\hat{\gamma}_{v}\left(l_{v}\right) \longrightarrow \hat{\gamma}(l)=q$. Also, again by Lemma 2.1 and Corollary 2.2, $\hat{\gamma}([0, l+\varepsilon]) \subset U$.

Let $\gamma=\hat{\gamma}_{\mid[0, l]}$. To end the proof we must show that $\gamma_{v} \longrightarrow \gamma$ in the $C^{0}$ topology on curves. Suppose the converse. Then one can find an open set $V \supset \gamma([0, l])$ such that, again after passing to a subsequence, for every $v$ there exists a $t_{v} \in\left[0, l_{v}\right]$ with $\gamma_{v}\left(t_{v}\right) \notin V$. Take an open $\hat{V}$ such that $V \subset \hat{V}$ and $\hat{\gamma}([0, l+\varepsilon]) \subset \hat{V}$. By (2.3), again passing to a subsequence if necessary, we have $\hat{\gamma}_{\nu}\left(\left[0, l_{\nu}\right]\right) \subset \hat{V}$ for every $\nu$. Thus $\gamma_{v}\left(t_{v}\right) \in \hat{V} \backslash V$ for every $\nu$. Passing to a subsequence again we may suppose that there exists a $t \in[0, l]$ such that $t_{v} \longrightarrow t$. By (2.3) $\gamma_{v}\left(t_{v}\right) \longrightarrow \gamma(t)$ and since $\hat{V} \backslash V$ is relatively closed in $\hat{V}$ we finally obtain $\gamma(t) \notin V$ which is a contradiction.

Corollary 2.3 Suppose that the fields $X_{0}, \ldots, X_{k}$ in (1.1) are linearly independent. Then the set $\mathcal{A}\left(q_{0}, U ; C\right)$ is closed with respect to $U$.

Proof Take a sequence $\left\{q_{\nu}\right\} \subset \mathcal{A}\left(q_{0}, U ; C\right)$ such that $q_{v} \longrightarrow q \in U$. For every $v$ there exists a trajectory $\gamma_{\nu}$ of (1.1) joining $q_{0}$ to $q_{\nu}$. The sequence $\left\{\gamma_{\nu}\right\}$ satisfies the assumptions of Proposition 2.2. Therefore, after passsing to a subsequence, $\gamma_{v} \longrightarrow \gamma$ in the $C^{0}$ topology on curves, where $\gamma$ is a trajectory of (1.1) that joins $q_{0}$ to $q$. In this way $q \in \mathcal{A}\left(q_{0}, U ; C\right)$ which ends the proof.

Now using [3] we have $c l_{U} \mathcal{A}\left(q_{0}, U ; C\right)=c l_{U} \mathcal{A}\left(q_{0}, U ; r i C\right)=c l_{U} \mathcal{A}\left(q_{0}, U\right.$; $r b C$ ) which together with Corollary 2.3 gives

Proposition 2.3 $\mathcal{A}\left(q_{0}, U ; C\right)=c l_{U} \mathcal{A}\left(q_{0}, U ; r i C\right)=c l_{U} \mathcal{A}\left(q_{0}, U ; r b C\right)$ for every $U \in \mathcal{O}$.

Now we strengthen our assumption; namely, up to the end of this section we assume that the fields $X_{0}, \ldots, X_{k}$ in (1.1) are linearly independent and such that Lie $_{q_{0}}\left\{X_{0}+\right.$ $\left.\sum_{j=1}^{k} u_{j} X_{j}: u \in r b C\right\}=T_{q_{0}} M$. This condition is open, so we will assume that all the sets from $\mathcal{O}$ are so small that 


$$
\forall U \in \mathcal{O} \quad \forall q \in U \quad \operatorname{Lie}_{q}\left\{X_{0}+\sum_{j=1}^{k} u_{j} X_{j}: u \in r b C\right\}=T_{q} M
$$

Recall that $U$ is a fixed element of $\mathcal{O}$. Introduce three other reachable sets in $U$ : $\mathcal{F}_{q_{0}}(C)$ (resp. $\mathcal{F}_{q_{0}}($ riC $), \mathcal{F}_{q_{0}}(r b C)$ ) is the reachable set from $q_{0}$ for the family of vector fields $\left\{X_{0}+\sum_{j=1}^{k} u_{j} X_{j}: u \in C\right\}$ (resp. for the family $\left\{X_{0}+\sum_{j=1}^{k} u_{j} X_{j}: u \in \operatorname{riC}\right\}$, $\left.\left\{X_{0}+\sum_{j=1}^{k} u_{j} X_{j}: u \in r b C\right\}\right)$. The set $\mathcal{F}_{q_{0}}(C)$ may be regarded as the set of endpoints of all trajectories of (1.1) that are generated by piecewise constant controls with values in $C$. Similar remarks apply to $\mathcal{F}_{q_{0}}(r i C), \mathcal{F}_{q_{0}}(r b C)$. Obviously $\mathcal{F}_{q_{0}}(C) \subset \mathcal{A}\left(q_{0}, U ; C\right), \mathcal{F}_{q_{0}}(r i C) \subset \mathcal{A}\left(q_{0}, U ; r i C\right), \mathcal{F}_{q_{0}}(r b C) \subset \mathcal{A}\left(q_{0}, U ; r b C\right)$.

Using again [3] (and Proposition 2.3) we have

$$
c l_{U} \mathcal{F}_{q_{0}}(r i C)=c l_{U} \mathcal{F}_{q_{0}}(r b C)=\mathcal{A}\left(q_{0}, U ; C\right)
$$

Because of (2.4) (cf. remark 1.1) we can apply Krener's theorem [10] for $\mathcal{F}_{q_{0}}(r b C)$ and $\mathcal{F}_{q_{0}}($ ri $C)$ to obtain

$$
\mathcal{F}_{q_{0}}(r i C) \subset c l_{U}\left(i n t \mathcal{F}_{q_{0}}(r i C)\right), \quad \mathcal{F}_{q_{0}}(r b C) \subset c l_{U}\left(i n t \mathcal{F}_{q_{0}}(r b C)\right) .
$$

Now (2.5), (2.6) give (1.3).

Next we will prove the first equality in (1.4). Clearly int $\mathcal{A}\left(q_{0}, U ;\right.$ riC $) \subset$ $\operatorname{int} \mathcal{A}\left(q_{0}, U ; C\right)$, so the reverse inclusion must be proven. Let $q \in \operatorname{int} \mathcal{A}\left(q_{0}, U ; C\right)$. There exists an open set $V$ such that $q \in V \subset \mathcal{A}\left(q_{0}, U ; C\right)$. Denote by $\mathcal{F}_{q}^{-}($ri $C)$ the reachable set from $q$ in $U$ for the family of vector fields $\left\{-X_{0}-\sum_{j=1}^{k} u_{j} X_{j}: u \in\right.$ riC . Again by Krener's theorem int $\mathcal{F}_{q}^{-}($riC $) \neq \varnothing$, thus int $\mathcal{F}_{q}^{-}($riC $) \cap V \neq \varnothing$. By the first equality in $(1.3)$, int $\mathcal{A}\left(q_{0}, U ; r i C\right)$ is dense in $\mathcal{A}\left(q_{0}, U ; C\right)$, so there is a point $q_{1}$ such that $q_{1} \in \operatorname{int} \mathcal{A}\left(q_{0}, U ;\right.$ riC $) \cap i n t \mathcal{F}_{q}^{-}(r i C) \cap V$. It follows that there exist admissible trajectories $\gamma_{1}, \gamma_{2}$ contained in $U$ such that $\gamma_{1}$ joins $q_{0}$ to $q_{1}, \gamma_{2}$ joins $q_{1}$ to $q$ and both are generated by controls with values in $r i C$. Also $\gamma_{1} \cup \gamma_{2}$ is contained in $\operatorname{int} \mathcal{A}\left(q_{0}, U ; r i C\right)$ by a well-kown fact saying that any admissible trajectory that starts from the interior of the reachable set cannot leave this interior. Summing up $q \in \operatorname{int} \mathcal{A}\left(q_{0}, U ;\right.$ riC $)$.

The equality int $\mathcal{A}\left(q_{0}, U ; C\right)=\operatorname{int} \mathcal{A}\left(q_{0}, U ; r b C\right)$ is proved analogously. Finally, observe that (1.5) follows directly from (1.3) and (1.4). Thus the proof of Theorem 1.1 is over.

\section{Proof of Proposition 1.1}

By Corollary 2.3 we need to prove only the second part. We start from the notion of so-called Goh curves. Let $H$ be a rank $m$ vector distribution on an $n$-dimensional manifold $M$. An absolutely continuous curve $\gamma:[a, b] \longrightarrow M$ is called horizontal if $\dot{\gamma}(t) \in H_{\gamma(t)}$ for almost every $t \in[a, b]$. Let $H^{\perp}$ denote the set of all such covectors $\lambda \in T^{*} M$ that $\langle\lambda, \xi\rangle=0$ for every $\xi \in H_{\pi(\lambda)}, \pi: T^{*} M \longrightarrow M$ being the canonical projection. $H^{\perp}$ is, in a natural way, a subbundle of rank $2 n-m$ of the 
cotangent bundle $T^{*} M$, and is called the annihilator of $H$. An absolutely continuous curve $\lambda:[a, b] \longrightarrow T^{*} M$ is called an abnormal biextremal if $\lambda([a, b]) \subset H^{\perp}, \lambda$ never intersects the zero section, and moreover $\Omega_{\lambda(t)}(\dot{\lambda}(t), \zeta)=0$ for almost every $t \in[a, b]$ and every $\zeta \in T_{\lambda(t)} H^{\perp}$; here by $\Omega$ we denote the restriction to $H^{\perp}$ of the standard symplectic form on $T^{*} M$. Now a horizontal curve $\gamma:[a, b] \longrightarrow M$ is said to be abnormal if there exists an abnormal biextremal $\lambda:[a, b] \longrightarrow T^{*} M$ such that $\gamma=\pi \circ \lambda$. Finally, an abnormal curve $\gamma:[a, b] \longrightarrow M$ is called a Goh curve, if there exists such an abnormal biextremal $\lambda:[a, b] \longrightarrow T^{*} M$ projecting onto $\gamma$, that for every $t \in[a, b]$ and for every smooth local sections $v, w$ of $H$ defined in a neighbourhood, say $G$, of $\gamma(t)$, we have $\langle\lambda(t),[v, w](\gamma(t))\rangle=0$ for each $t$ such that $\gamma(t) \in G$. According to [4] Goh curves do not exist for generic distributions of rank $m \geq 3$.

Now we pass to the proof of the second part of Proposition 1.1 Fix a $U \in \mathcal{O}$. By virtue of the above remark it is enough to show that every admissible trajectory $\gamma:[0, T] \longrightarrow U$ which is generated by a control $u:[0, T] \longrightarrow r i C$, and is such that $\gamma([0, T]) \subset \tilde{\partial} \mathcal{A}\left(q_{0}, U ; C\right)$ is a Goh curve. Admissible curves that start from $q_{0}$ and are contained in $\tilde{\partial} \mathcal{A}\left(q_{0}, U ; C\right)$ are called geometrically optimal curves. Clearly, $\gamma$ is geometrically optimal, and moreover, in view of our assumption imposed on $u$, one can suppose that it is geometrically optimal with respect to the set of control parameters being equal to $\mathrm{riC}$. Now we are going to write down necessary conditions for geometric optimality following from the Pontriagin maximum principle-see e.g. [1] (we agreed to use $L^{2}$ controls, however, $C$ is bounded so our controls are in fact $L^{\infty}$ and Pontriagin maximum principle is applicable).

To be more precise, for each $\alpha=0, \ldots, k$ denote by $h_{\alpha}$ the function $h_{\alpha}: T^{*} U \longrightarrow$ $\mathbb{R}$ defined by $h_{\alpha}(q, p)=\left\langle p, X_{\alpha}(q)\right\rangle$. For every $u \in C$ let us also set $h_{u}=h_{0}+$ $\sum_{j=1}^{k} u_{j} h_{j}$. Now the maximum principle asserts that if a curve $\gamma:[0, T] \longrightarrow U$, generated by $u:[0, T] \longrightarrow r i C$, is geometrically optimal, then there exists an absolutely continuous curve $\lambda:[0, T] \longrightarrow T^{*} U, \lambda(t) \in T_{\gamma(t)}^{*} U \backslash\{0\}$ for every $t \in[0, T]$, such that (i) $\dot{\lambda}(t)=\overrightarrow{h_{0}}(\lambda(t))+\sum_{j=1}^{k} u_{j}(t) \overrightarrow{h_{j}}(\lambda(t))$ a.e. on [0,T]; (ii) $h_{u(t)}(\lambda(t))=$ $\max _{v \in r i C} h_{v}(\lambda(t))$ a.e. on $[0, T]$; (iii) $h_{u(t)}(\lambda(t))=0$ on $[0, T]$. By $\overrightarrow{h_{\alpha}}$ we denoted the Hamiltonian vector field corresponding to $h_{\alpha}$.

Now (ii) implies that $h_{j}(\lambda(t))=0$ for $j=1, \ldots, k$ and every $t \in[0, T]$. This, together with (iii), gives $h_{0}(\lambda(t))=0$ for every $t \in[0, T]$ which means $\lambda([0, T]) \subset$ $H^{\perp}$, where $H=\operatorname{Span}\left\{X_{0}, \ldots, X_{k}\right\}$. Moreover, by definition of $\overrightarrow{h_{\alpha}}$,s,

$$
\Omega(\zeta, \dot{\lambda}(t))=d_{\lambda(t)} h_{0}(\zeta)+\sum_{j=1}^{k} u_{j}(t) d_{\lambda(t)} h_{j}(\zeta)
$$

which implies $\Omega(\zeta, \dot{\lambda}(t))=0$ for every $\zeta \in T_{\lambda(t)} H^{\perp}$. Thus we conclude that $\gamma$ is an abnormal curve.

Next, using [1], $\gamma$ satisfies the Goh condition, which in our case reads

$$
\left\langle\lambda(t),\left[X_{i}, X_{j}\right](\gamma(t))\right\rangle=0, \quad i, j=1, \ldots, k, t \in[0, T] .
$$


Differentiating the equation $\left\langle\lambda(t), X_{j}(\gamma(t))\right\rangle=0, j=1, \ldots, k$, with respect to $t$ on the interval $[0, T]$ one obtains

$$
0=\frac{d}{d t}\left\langle\lambda(t), X_{j}(\gamma(t))\right\rangle=\left\langle\lambda(t),\left[X_{j}, X_{0}\right](\gamma(t))\right\rangle+\sum_{i=1}^{k} u_{i}(t)\left\langle\lambda(t),\left[X_{j}, X_{i}\right](\gamma(t))\right\rangle
$$

which, using (3.1), gives

$$
\left\langle\lambda(t),\left[X_{\alpha}, X_{\beta}\right](\gamma(t))\right\rangle=0, \quad \alpha, \beta=0,1, \ldots, k, t \in[0, T]
$$

The proof of Proposition 1.1 is finished.

It is easy to give an example of an affine control system where $\mathcal{A}\left(q_{0}, U ; r i C\right)$ is not open. To this end take $a, b \in \mathbb{R}$ such that $a<0<b$, and consider a system

$$
\dot{q}=\frac{\partial}{\partial x}+\frac{1}{2} y^{2 k} \frac{\partial}{\partial z}+u\left(\frac{\partial}{\partial y}-\frac{1}{2} x y^{2 k-1} \frac{\partial}{\partial z}\right)
$$

where $k$ is a positive integer and $u \in C=[a, b]$. Using the same argument as in [7] one can see that the curve $\gamma(t)=(t, 0,0)$ (which is generated by the null control $u(t)=0)$ is the only, up to a change of parameter, admissible curve joining $(0,0,0)$ to a point $(0,0, T)$ for any $T>0$. Now (1.4) yields that $\gamma([0, T]) \subset \tilde{\partial} \mathcal{A}\left(0, \mathbb{R}^{3} ; C\right)$, and what follows $\mathcal{A}\left(0, \mathbb{R}^{3} ; \mathrm{riC}\right)$ is not open.

\section{Remarks}

First of all let us note that throughout the paper we do not assume completeness of admissible trajectories for positive times.

Now we make some comments on a system $\mathcal{O}$ of neighbourhoods of $q_{0}$. It is intuitively obvious that (1.3) does not hold for every neighbourhood of $q_{0}$. To construct an example of such a "bad" neighbourhood let us consider the control affine system determined by the Heisenberg sub-Lorentzian metric on $\mathbb{R}^{3}$ (see [6]). In this case, as it was explained in the introduction, $C=[-1,1]$. Take a number $\delta>0$ and define a convex neighbourhood of the origin

$$
U_{\delta}=\left\{(x, y, z):-\frac{1}{2} x-\delta<y<-\frac{1}{2} x+\delta, \frac{1}{2} x-\delta<y<\frac{1}{2} x+\delta,-\delta^{2}<z<\delta^{2}\right\} .
$$

Let $\delta_{n}$ be an arbitrary sequence of real numbers such that $0<\delta_{n} \nearrow \frac{4}{3} \delta$. Now, by [6], it is clear that $q_{n}=\left(\delta_{n}, 0, \frac{1}{4} \delta_{n}^{2}\right) \in \tilde{\partial} \mathcal{A}\left(0, U_{\delta} ;[-1,1]\right)$ while $q=\lim q_{n} \notin$ $\mathcal{A}\left(0, U_{\delta} ;[-1,1]\right)$, hence $\mathcal{A}\left(0, U_{\delta} ;[-1,1]\right)$ is not closed with respect to $U_{\delta}$ for every $\delta>0$, and consequently (1.3) is no longer true for a $U_{\delta}$ 's. At the same time, let us note that $\mathcal{A}\left(0, \mathbb{R}^{3} ;[-1,1] ; \leq T\right) \cap U_{\delta}$ is closed with respect to $U_{\delta}$ for every $T>0$, as it follows from theorems mentioned in the introduction.

In order to prove Theorem 1.1, it is in fact sufficient to consider small enough normal neighbourhoods of $q_{0}$ but such an approach is not very constructive and requires entering into the sub-Lorentzian geometry details. From practical reasons it is simpler 
to construct the elements of $\mathcal{O}$ as presented in sect. 2, i.e. for a point $q_{0}$ find a function $\varphi$, next take a sufficiently small starshaped neighbourhood of $q_{0}$ so that (2.1) holds, and finally modify the boundary $\partial U$ in such a way that $\mathcal{A}\left(q_{0}, \hat{U} ; C\right) \cap \partial U$ is a level set of $\varphi$. Let us emphasize that we do not need to know the set $\mathcal{A}\left(q_{0}, \hat{U} ; C\right)$ - for small $U$ 's it is easy to estimate the region in which $\mathcal{A}\left(q_{0}, \hat{U} ; C\right)$ is contained.

Next remark concerns assumptions imposed on the distribution $H=\operatorname{Span}\left\{X_{0}\right.$, $\left., \ldots, X_{k}\right\}$. We saw in remark 1.1 that under assumptions of Theorem $1.1 H$ is bracket generating. Now we will explain what can be said about (1.3), (1.4) and (1.5) if $H$ is not bracket generating. Our reasoning is based on [13]. So let $\hat{H}$ be the smallest integrable distribution containing $H$; since $H$ is not bracket generating, $\hat{H}$ is not equal to the whole $T M$. Our $M$ is foliated by leaves of $\hat{H}$, i.e. $M$ is the union of integral submanifolds of $\hat{H}$. Let $L$ be the (unique) leaf that passes through $q_{0}$. Obviously, reachable sets from $q_{0}$ for the system (1.1) are now contained in $L$. Note here that, in general, $L$ is an immersed submanifold, so the appropriate topology to consider is the manifold topology of $L$ (which may be stronger than the topology induced by $M$ ). Now (1.3), (1.4) and (1.5) remain valid if we modify assumptions of Theorem 1.1 as follows. At first, $\mathcal{O}$ is the fundamental system of neighbourhoods of $q_{0}$ on $L$. Secondly, we should replace $T_{q_{0}} M$ by $T_{q_{0}} L$ in the right hand side of (1.2). Finally, the operations $c l_{U}$ and $\tilde{\partial}$ must be taken with respect to the manifold topology of $L$.

At the end let us emphasize the application of the sub-Lorentzian geometry methods to the study of control affine systems. Sub-Lorentzian metrics can play similar auxiliary role in the investigation of control affine systems as, for instance, Riemannian metrics do in many problems in differential topology.

Open Access This article is distributed under the terms of the Creative Commons Attribution Noncommercial License which permits any noncommercial use, distribution, and reproduction in any medium, provided the original author(s) and source are credited.

\section{References}

1. Agrachev, A., Sakchov, Y.: Control Theory from Geometric Viewpoint, Encyclopedia of Mathematical Science, vol. 87. Springer, Berlin (2004)

2. Bismut, J.-M.: Large Deviations and the Malliavin Calculus. Birkhäuser, Boston (1984)

3. Bressan, A., Piccoli, B.: Introduction to the mathematical theory of control. AIMS Series Appl. Math., vol. 2 (2007)

4. Chitour, Y., Jean, F., Trélat, E.: Genericity result for singular curves. J. Diff. Geom. 73(1) (2006)

5. Grochowski, M.: Geodesics in the sub-Lorentzian Geometry. Bull. Pol. Acad. Sci. 50(2) (2002)

6. Grochowski, M.: Reachable Sets for the Heisenberg sub-Lorentian Metric on $\mathbb{R}^{3}$. An estimate for the distance function. J. Dyn. Control Syst. 12(2) (2006)

7. Grochowski, M.: Properties of reachable sets in the sub-Lorentzian geometry. J. Geom. Phys. 59, 885-900 (2009)

8. Hermès, H., Lasalle, J.P.: Functional Analysis and Time Optimal Control, Mathematics in Sciences and Engineering, vol. 56. Academic Press, New York (1969)

9. Jurdjevich, V.: Geometry Control Theory. Cambridge University Press, Cambridge (1996)

10. Krener, A.J.: A generalization of chow's theorem and the bang-bang theorem to nonlinear control problem. SIAM J. Control Optim. 15(2) February (1977)

11. Lojasiewicz, S.: An Introduction to the Theory of Real Functions. Wiley, Chichester (1988)

12. Rockaffelar, R.T.: Convex Analysis. Princeton University Press, Princeton (1972)

13. Sussmann, H.: Orbits of families of vector fields and integrability of distributions. Trans. Am. Math. Soc. 180, 171-188 (1973) 